\title{
Novel synergistic antibiofilm combinations for salvage of infected catheters
}

Correspondence Mohan Venkatesh mohanv@bcm.edu

Received 21 January 2009 Accepted 20 March 2009

\author{
Mohan Venkatesh, ${ }^{1}$ Liang Rong, ${ }^{1}$ Issam Raad ${ }^{2}$ and James Versalovic ${ }^{3}$ \\ ${ }^{1}$ Section of Neonatology, Department of Pediatrics, Texas Children's Hospital \& Baylor College of \\ Medicine, Houston, TX 77030, USA \\ ${ }^{2}$ Department of Infectious Diseases, MD Anderson Cancer Center, Houston, TX 77030, USA \\ ${ }^{3}$ Department of Pathology, Texas Children's Hospital \& Baylor College of Medicine, Houston, TX \\ 77030, USA
}

\begin{abstract}
Biofilms on catheters are responsible for catheter-related bloodstream infections (CRBSIs), which cause significant mortality and morbidity. Antimicrobial catheter-lock solutions may salvage precious catheters by eradicating biofilms. Staphylococcus epidermidis and Candida albicans are frequently isolated organisms in CRBSls. We evaluated $N$-acetylcysteine (NAC), EDTA, ethanol and talactoferrin (TLF) individually and in combination with antibiotics against biofilms of $S$. epidermidis and C. albicans to identify effective catheter-lock solutions. Minimum biofilmeradication concentrations causing $50 \%$ inhibition $\left(\right.$ MBEC $_{50}$ ) for EDTA, NAC, ethanol and TLF were determined against biofilms of $S$. epidermidis and $C$. albicans formed on 96-well microtitre plates. Biomass, mean thickness and viability of $S$. epidermidis and $C$. albicans biofilms were evaluated after exposure to $\mathrm{MBEC}_{50}$ concentrations of EDTA, NAC, ethanol and TLF. Antimicrobial combinations of EDTA, NAC, ethanol and TLF with nafcillin, vancomycin, fluconazole and amphotericin $B$ were evaluated systematically for synergy using combination indices (Cls). EDTA, NAC, ethanol and TLF significantly reduced biofilm biomass and mean thickness $(P<0.05$, one-way ANOVA) of monomicrobial and polymicrobial biofilms as evaluated by confocal microscopy. Cls evaluated at equipotency ratios, and 50, 75 and $90 \%$ effects, showed that EDTA, NAC, ethanol and TLF were synergistic $(\mathrm{Cl}<1)$ with antibiotics (with few exceptions) against biofilms of $S$. epidermidis and C. albicans. EDTA, NAC, ethanol and TLF inhibit monomicrobial and polymicrobial biofilms of neonatal strains of S. epidermidis and C. albicans, and are synergistic with antibiotics. Catheter-lock solutions of EDTA, NAC and ethanol alone or in combination with antibiotics may be used to salvage infected catheters, which will directly impact on patient morbidity and health-care costs.
\end{abstract}

\section{INTRODUCTION}

Biofilms on catheters are responsible for about $85 \%$ of catheter-related bloodstream infections (CRBSIs) in intensive-care units, and cause significant mortality and morbidity (Console et al., 2007; Donelli, 2006; Pawar et al., 2004). A single episode of CRBSI is estimated to cost as much as $\$ 56167$ ( $£ 38180$ ) (Dimick et al., 2001; Rello et al., 2000). Removal of medical devices is often required to eradicate biofilm-associated infections (Gandelman et al.,

Abbreviations: $\mathrm{Cl}$, combination index; CLSI, Clinical and Laboratory Standards Institute; CRBSI, catheter-related bloodstream infection; $E D$, effective dose; EPS, exopolysaccharide; $M_{B E C}{ }_{50}$, minimum biofilm eradication concentration causing 50\% inhibition; NAC, $\mathrm{N}$-acetylcysteine; TLF, talactoferrin; XTT, 2, 3-bis(2-methoxy-4 nitro-5sulfophenyl)-2H tetrazolium-5-carboxanilide.

Figures showing biofilm viability data and tables of $\mathrm{Cls}$ are available as supplementary data with the online version of this paper.
2007). The most frequent organisms causing CRBSIs are Staphylococcus epidermidis, Staphylococcus aureus and Candida species (Raad \& Hanna, 2002), and these microbes have been isolated together in biofilm-related polymicrobial infections (Karlowicz et al., 2000). The increased mortality and morbidity due to polymicrobial infections makes it imperative to evaluate new treatment modalities against polymicrobial biofilms (Faix \& Kovarik, 1989; McKenzie, 2006). Hence, we evaluated monomicrobial and polymicrobial biofilms in our experimental studies.

Antimicrobial agents alone or in combination locked in the lumen of catheters for varying time periods have been effective against biofilms on intravascular catheters (Anaissie et al., 1995; Raad et al., 2003, 2007a, b) and have been recommended to prevent CRBSIs (O'Grady et al., 2002). We sought to evaluate antibiofilm agents individually and in combination with antibiotics against clinical 
isolates of organisms to emphasize the utility of antimicrobial-lock combinations in the salvage of infected catheters.

The emergence of alarming levels of antibiotic resistance urged us to look at other non-antibiotic strategies against CRBSIs (Levy, 1998). We evaluated $N$-acetylcysteine (NAC), EDTA, ethanol and talactoferrin (TLF) (human recombinant lactoferrin). NAC is used as a mucolytic and may disrupt or prevent exopolysaccharide (EPS) formation in biofilms (Perez-Giraldo et al., 1997). EDTA, a chelator of calcium and magnesium, and an anticoagulant, inhibits planktonic Staphylococcus and Candida species (Gil et al., 1994; Root et al., 1988). Ethanol has been shown to be effective in eradicating monomicrobial biofilms without altering the mechanical properties of silicone or polyurethane catheters (Crnich et al., 2005; Raad et al., 2007a). Lactoferrin is an iron-binding glycoprotein, which is naturally present in human glandular secretions (milk, tears and saliva), and has broad-spectrum antimicrobial activity against bacteria and fungi (Valenti \& Antonini, 2005).

The effects of NAC, EDTA, ethanol or lactoferrin (TLF) have not been evaluated against polymicrobial biofilms of S. epidermidis and Candida albicans. Therefore, we evaluated NAC, EDTA, ethanol and TLF on monomicrobial and polymicrobial biofilms of $C$. albicans and $S$. epidermidis. Interactions of these agents with antibiotics in combination with nafcillin, vancomycin, fluconazole and amphotericin B were evaluated for synergy. Novel antimicrobial strategies specifically targeting biofilms with synergistic antibiotic combinations are urgently needed to reduce CRBSIs and improve clinical outcomes (Furuya \& Lowy, 2003).

\section{METHODS}

Organisms. Clinical isolates of S. epidermidis (ATCC 55133, H100 and S101) and C. albicans (ATCC 32354 and MYA 4441) were used (two strains of $S$. epidermidis and one strain of $C$. albicans were isolated from septic neonates).

Antimicrobial agents. Antimicrobial agents were prepared as stock solutions as recommended by the Clinical and Laboratory Standards Institute (CLSI) wherever appropriate (CLSI, 2002, 2003, 2007). Dilutions were performed in the growth media appropriate for the organism and the following agents were used: (i) NAC (USB) $\mathrm{pH}$ adjusted to 7 and evaluated in twofold serial dilutions from 32 to $0.5 \mathrm{mg} \mathrm{ml}^{-1}$; (ii) disodium EDTA (Sigma-Aldrich), $\mathrm{pH}$ adjusted to 7 and evaluated in twofold serial dilutions from 32 to $0.5 \mathrm{mg} \mathrm{ml}^{-1}$; (iii) ethanol (AAPER Alcohol and Chemical) evaluated in twofold serial dilutions from 100 to $3 \%$; (iv) recombinant human lactoferrin (TLF $\alpha$ ) (Agennix), evaluated in twofold serial dilutions from 66.4 to $0.125 \mathrm{mg} \mathrm{ml}^{-1}$; (v) fluconazole (Sigma-Aldrich), evaluated in twofold serial dilutions from 128 to $0.125 \mu \mathrm{g} \mathrm{ml}^{-1}$; (vi) amphotericin B (Sigma-Aldrich) evaluated in twofold serial dilutions from 128 to $0.125 \mu \mathrm{g} \mathrm{ml}^{-1}$; (vii) vancomycin (USB) evaluated in twofold serial dilutions from 64 to $0.5 \mu \mathrm{g} \mathrm{ml}^{-1}$; (viii) nafcillin (Sigma-Aldrich) evaluated in twofold serial dilutions from 64 to $0.5 \mu \mathrm{g} \mathrm{ml}^{-1}$.

Biofilms. Biofilms were formed in 96-well microtitre plates by adding $100 \mu \mathrm{l} \mathrm{S}$. epidermidis or C. albicans at approximately $10^{6}$ c.f.u. $\mathrm{ml}^{-1}$ individually or $50 \mu \mathrm{l}$ of both (for polymicrobial biofilms) in RPMI 1640 medium ( $\mathrm{pH} \mathrm{7}$ ) and incubating for $24 \mathrm{~h}$ at $35^{\circ} \mathrm{C}$. Biofilm formation was confirmed by light microscopy and 2, 3-bis(2methoxy-4 nitro-5-sulfophenyl)-2H tetrazolium-5-carboxanilide (XTT) reduction assay as reported by other investigators (Cerca et al., 2004, 2005; Ramage \& Lopez-Ribot, 2005).

Evaluation of minimum biofilm eradication concentration causing $\mathbf{5 0} \%$ inhibition (MBEC ${ }_{50}$ ) of single antibiofilm agents. Evaluation of the antimicrobial susceptibility of biofilms to antimicrobial agents was performed in duplicate using adapted CLSI guidelines (Domingue et al., 1994; Ramage \& Lopez-Ribot, 2005). Minimum biofilm eradication concentration was defined as the minimum concentration of the antibiotic needed to eradicate $50 \%$ of the biofilm - the MBEC $_{50}$. Growth and sterility controls on the same microtitre plate were used for comparison. The biofilms in the microtitre plates were exposed to the antibiofilm agent for $20 \mathrm{~h}$ (S. epidermidis) or $48 \mathrm{~h}$ (C. albicans). Inhibition end point was assessed by XTT reduction assay as described by Hawser et al. (1998). Briefly, XTT $\left(0.5 \mathrm{~g}^{-1}\right.$ in Ringer's lactate) and menadione $(1 \mu \mathrm{M})$ were added to washed biofilms, incubated in the dark for $2 \mathrm{~h}$ at $37^{\circ} \mathrm{C}$ and the colorimetric change in the supernatant was measured in a microtitre plate reader as the absorbance at $490 \mathrm{~nm}$.

Evaluation of biofilm architecture by confocal laser scanning microscopy. Biofilms of C. albicans ATCC 32354 and S. epidermidis ATCC 55133 were formed on 4-well optical bottom glass slides. These biofilms were exposed to NAC, disodium EDTA and TLF at $8 \mathrm{mg} \mathrm{ml}$, ethanol at $12.5 \%$ or growth medium (control) for $24 \mathrm{~h}$. The biofilms were washed and stained with LIVE/DEAD stain and examined using a Zeiss meta confocal microscope. Serial sections in the $x y$ plane were obtained at $1 \mu \mathrm{m}$ intervals along the $z$-axis, and the $z$-stack image was analysed by COMSTAT 2 software for biomass and mean thickness (Heydorn et al., 2000). Biofilms exposed to the antimicrobial agents were compared with the controls by one-way ANOVA and statistical significance was assumed at $P<0.05$.

Evaluation of biofilm viability after exposure to antibiofilm agents. Biofilms of S. epidermidis, C. albicans or both were formed in 96-well microtitre plates and were exposed to EDTA, NAC and TLF at $16 \mathrm{mg} \mathrm{ml}^{-1}$, ethanol at $12.5 \%$ or medium (control). At $0,24,48$ and $72 \mathrm{~h}$, the biofilms were scraped, sonicated ten times for $10 \mathrm{~s}$ and plated in serial dilutions. For polymicrobial biofilms, the dilutions were plated in trypticase soy agar with amphotericin B ( $S$. epidermidis) and in Sabouraud dextrose agar with oxacillin ( $C$. albicans) enabling the determination of the relative contributions of the two organisms to biofilm composition and relative viability after antimicrobial susceptibility testing. Evaluations were done in triplicate and the experiment was repeated at least twice (at least six evaluations at any time point for each intervention). Results were analysed by two-way ANOVA using the Graph Pad Prism (version 4) statistical software.

Evaluation of antimicrobial combinations for synergy. EDTA, NAC, ethanol and TLF were combined with vancomycin and nafcillin against S. epidermidis, and with amphotericin B and fluconazole against C. albicans. Susceptibilities were evaluated in an 8-well by 8well chequerboard format in 96-well micro-titre plates. Antibiofilm agents were prepared in twofold serial dilutions across rows and columns, and inhibitory end points were assessed by the XTT reduction assay similar to evaluation of $\mathrm{MBEC}_{50}$ for single agents. Inhibitory effects at equipotent drug-dose ratios (ratios of $\mathrm{MBEC}_{50}$ ) of the combinations were determined. The median effects method described by Chou and colleagues was used to study interactions in drug combinations by the calculation of combination indices (CIs) (Chou \& Talalay, 1984; Chou, 2006). 
The median effects principle is given by the equation: $f_{\mathrm{a}} / f_{\mathrm{u}}=\left(D / D_{\mathrm{m}}\right)^{m}$, where $f_{\mathrm{a}}$ is the fraction affected by the drug, $f_{\mathrm{u}}$ is the fraction unaffected $\left(f_{\mathrm{u}}=1-f_{\mathrm{a}}\right), D$ is the concentration of the drug and $D_{\mathrm{m}}$ is the median effect dose [the dose of the drug that produces $50 \%$ of the effect, i.e. the effective dose $\left._{50}\left(\mathrm{ED}_{50}\right)\right]$. The co-efficient of the doseeffect relationship is given by ' $m$ ', and $m=1,>1$ and $<1$ indicates hyperbolic, sigmoidal and flat-sigmoidal dose-effect curves, respectively.

The CI is given by the equation: $\mathrm{CI}=(D)_{1} /\left(D_{\mathrm{x}}\right)_{1}+(D)_{2} /\left(D_{\mathrm{x}}\right)_{2}$, where $(D)_{1}$ and $(D)_{2}$ are doses of drug 1 and drug 2 in combination, and $\left(D_{\mathrm{x}}\right)_{1}$ and $\left(D_{\mathrm{x}}\right)_{2}$ are doses of drug 1 and drug 2 that produce $x \%$ effect when used alone. A CI $<1$ indicates synergy, CI $>1$ antagonism and $\mathrm{CI}=1$, an additive effect. Multiple drug dose-effect calculations were performed using Calcusyn software (Biosoft) (http://www.biosoft. $\mathrm{com} / \mathrm{w} /$ calcusyn.htm) with constant ratios of drug combinations.

\section{RESULTS AND DISCUSSION}

\section{MBEC $_{50}$}

Antimicrobial susceptibility testing for biofilms of $C$. albicans and $S$. epidermidis have not been standardized and hence we adapted the CLSI guidelines for planktonic MIC testing to our biofilm experiments (CLSI, 2002, 2003, 2007). MBEC $_{50}$ values for NAC, disodium EDTA, ethanol and TLF were comparable to results from other investigators and are shown in Table 1 (Bachmann et al., 2003; Leitch \& Willcox, 1999a; Marchese et al., 2003; Olofsson et al., 2003; Percival et al., 2005).

\section{Evaluation of biofilms by confocal imaging microscopy}

Confocal microscopy is frequently used in evaluating biofilm architecture and is preferred over electron microscopy where the embedding process dehydrates the biofilm specimen (Heydorn et al., 2000). We found that NAC, EDTA and TLF at $8 \mathrm{mg} \mathrm{ml}^{-1}$ and ethanol at $12.5 \%$ significantly decreased biofilm biomass, and mean thickness of monomicrobial and polymicrobial biofilms of $S$. epidermidis ATCC 55133 and C. albicans ATCC 32354 (Figs 1, 2 and 3) compared to controls exposed to growth media $(P<0.05$, one-way ANOVA). Interestingly we noticed that the concentrations of NAC, EDTA, ethanol
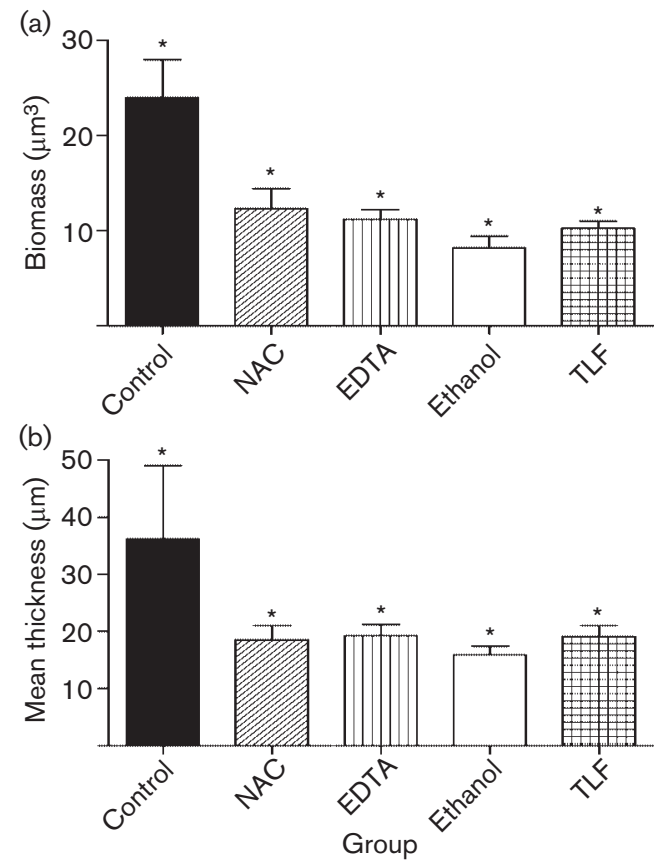

Fig. 1. Antibiofilm agents decrease biomass and thickness of $S$. epidermidis ATCC 55133 biofilms. S. epidermidis ATCC 55133 biofilms grown in optical bottom glass slides were exposed to NAC, EDTA and TLF at $8 \mathrm{mg} \mathrm{ml}^{-1}$, ethanol at $12.5 \%$ or growth medium (control) for $24 \mathrm{~h}$. Biomass (a) and mean thickness (b) were evaluated by confocal microscopy and data are presented as means $\pm S D$. NAC, EDTA, ethanol and TLF significantly decreased biofilm biomass and mean thickness compared to the controls $\left({ }^{*} P<0.05\right.$, one-way ANOVA).

and TLF required for inhibiting polymicrobial biofilms of S. epidermidis and C. albicans were at least twofold lower than the $\mathrm{MBEC}_{50}$ for monomicrobial biofilms. Dissemination of cell clusters in catheter-associated biofilms may occur after it reaches a critical mass, and reduction of biomass and mean thickness may be clinically significant in decreasing the incidence of CRBSIs (Stoodley et al., 2001).

Table 1. $M_{B E C}$ for EDTA, NAC, ethanol and TLF against monomicrobial and polymicrobial biofilms

\begin{tabular}{|c|c|c|c|c|c|c|c|c|}
\hline Organism & $\begin{array}{c}\text { NAF } \\
\left(\mu \mathrm{g} \mathrm{ml}^{-1}\right)\end{array}$ & $\begin{array}{c}\text { VAN } \\
\left(\mu \mathrm{g} \mathrm{ml}^{-1}\right)\end{array}$ & $\begin{array}{c}\mathrm{AMB} \\
\left(\mu \mathrm{g} \mathrm{ml}^{-1}\right)\end{array}$ & $\begin{array}{c}\text { FLC } \\
\left(\mu \mathrm{g} \mathrm{ml}^{-1}\right)\end{array}$ & $\begin{array}{c}\text { EDTA } \\
\left(\mathrm{mg} \mathrm{ml}^{-1}\right)\end{array}$ & $\begin{array}{c}\mathrm{NAC} \\
\left(\mathrm{mg} \mathrm{ml}^{-1}\right)\end{array}$ & $\begin{array}{c}\text { Ethanol } \\
(\%)\end{array}$ & $\begin{array}{c}\text { TLF } \\
\left(\mathrm{mg} \mathrm{ml}^{-1}\right)\end{array}$ \\
\hline S. epidermidis ATCC 55133 & 8 & 8 & - & - & 4 & 8 & 12.5 & 4 \\
\hline S. epidermidis $\mathrm{H} 100$ & 8 & 16 & - & - & 1 & 1 & 12.5 & 8 \\
\hline S. epidermidis $\mathrm{S} 101$ & 32 & 32 & - & - & 4 & 4 & 12.5 & 8 \\
\hline C. albicans ATCC 32354 & - & - & 64 & 64 & 32 & 32 & 12.5 & 33.2 \\
\hline C. albicans ATCC MYA 4441 & - & - & 32 & 32 & 16 & 32 & 12.5 & 66.4 \\
\hline $\begin{array}{l}\text { Polymicrobial biofilm of S. epidermidis ATCC } \\
55133 \text { and C. albicans ATCC } 32354\end{array}$ & - & - & - & - & 0.25 & 2 & 6 & 4 \\
\hline
\end{tabular}

AMB, Amphotericin B; FLC, fluconazole; NAF, nafcillin; VAN, vancomycin. 
(a)

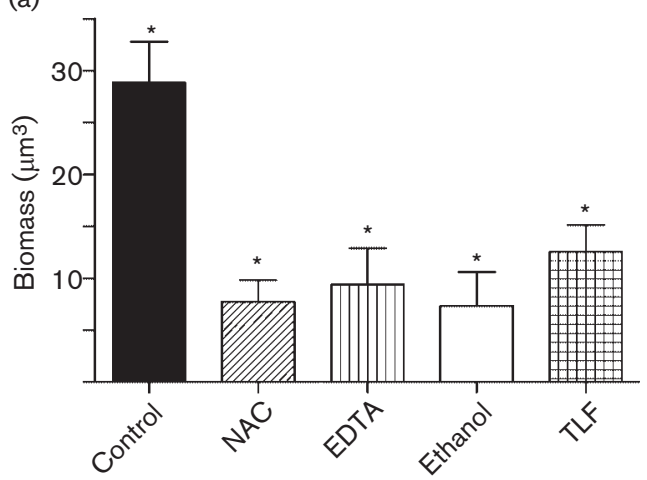

(b)

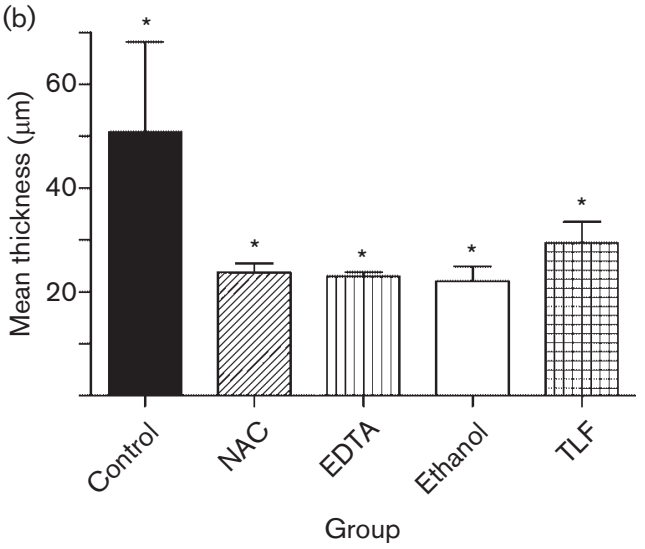

Fig. 2. Antibiofilm agents decrease biomass and thickness of $C$. albicans ATCC 32354 biofilms. C. albicans ATCC 32354 biofilms grown in optical bottom glass slides were exposed to NAC, EDTA and TLF at $8 \mathrm{mg} \mathrm{ml}^{-1}$, ethanol at $12.5 \%$ or growth medium (control) for $24 \mathrm{~h}$. Biomass (a) and mean thickness (b) were evaluated by confocal microscopy and data are presented as means $\pm S D$. NAC, EDTA, ethanol and TLF significantly decreased biofilm biomass and mean thickness compared to the controls $\left({ }^{\star} P<0.05\right.$, one-way ANOVA).

\section{Evaluation of the viability of polymicrobial biofilms after exposure to antibiofilm agents}

We evaluated polymicrobial biofilms of S. epidermidis and C. albicans, because polymicrobial infections of the above organisms are seen in patients with intravascular catheters (Faix \& Kovarik, 1989; Kaufman \& Fairchild, 2004; Noyola et al., 2001) but have not been adequately studied. In polymicrobial biofilms of S. epidermidis ATCC 55133 and C. albicans ATCC 32354, both organisms contributed proportionally to the biofilm at 24,48 and $72 \mathrm{~h}$ (Fig. 4a). Viability of S. epidermidis ATCC 55133 was significantly decreased by EDTA and ethanol at 24, 48 and $72 \mathrm{~h}$, by NAC at only 24 and $48 \mathrm{~h}$ and not by TLF (Fig. $4 \mathrm{~b}$ ). Viability of C. albicans ATCC 32354 was significantly reduced only by ethanol compared to the control at 24,48 and $72 \mathrm{~h}$. Other agents (EDTA, NAC and TLF) did not reduce the viability of $C$. albicans (Fig. 4c). (a)

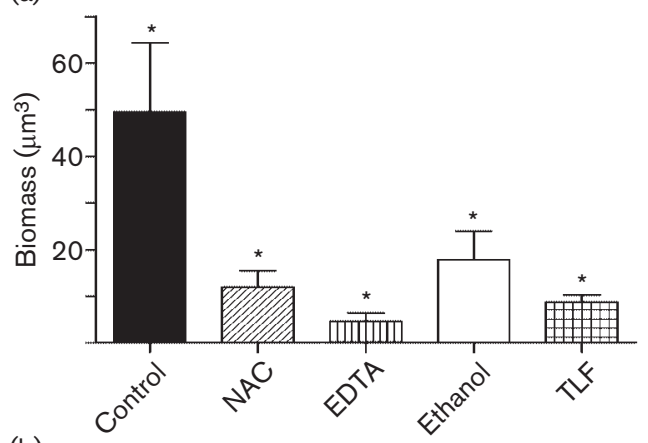

(b)

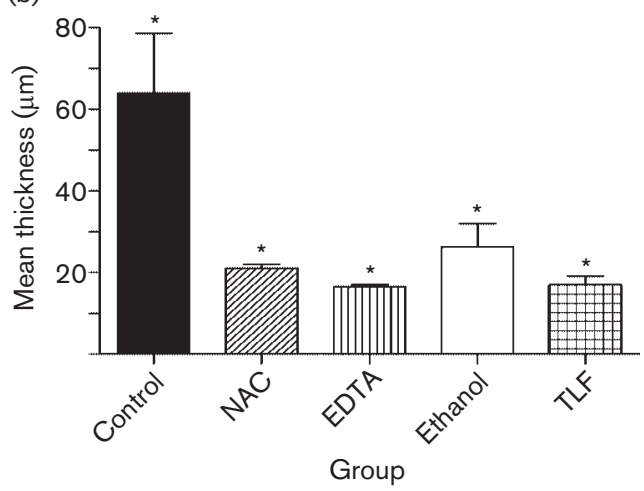

Fig. 3. Antibiofilm agents decrease biomass and thickness of polymicrobial biofilms. Polymicrobial biofilms of $S$. epidermidis ATCC 55133 and C. albicans ATCC 32354 grown in optical bottom glass slides were exposed to NAC, EDTA and TLF at $8 \mathrm{mg}$ $\mathrm{ml}^{-1}$, ethanol at $12.5 \%$ or growth medium (control) for $24 \mathrm{~h}$. Biomass (a) and mean thickness (b) were evaluated by confocal microscopy and data are presented as means \pm SD. NAC, EDTA, ethanol and TLF significantly decreased biofilm biomass and mean thickness compared to the controls ( ${ }^{*} P<0.05$, one-way ANOVA).

Ethanol significantly reduced biofilm viability in polymicrobial biofilms and monomicrobial biofilms of all $C$. albicans strains, and monomicrobial biofilms of two strains of S. epidermidis after 24, 48 and $72 \mathrm{~h}$ of exposure (Supplementary Figs S1 and S2 available with the online journal). Inconsistent efficacy of ethanol against $S$. epidermidis in our experiments may be due to reduced concentrations of ethanol used in these experiments. Other investigators using higher concentrations of ethanol $(>12.5 \%)$ have demonstrated efficacy of ethanol against biofilms composed of S. aureus or Candida parapsilosis. In conjunction with minocycline and EDTA, ethanol eradicated biofilm growth on catheter discs (Raad et al., 2007a). Chambers et al. (2006) reported that ethanol (70\% v/v) was effective against monomicrobial biofilms of $C$. albicans, Pseudomonas aeruginosa or Klebsiella pneumoniae grown in microtitre plates after exposure to ethanol for $4 \mathrm{~h}$. In early clinical studies, catheter-lock solutions containing $70 \%$ ethanol were effective in preventing or treating catheter infections without adverse effects (Broom et al., 2008; Mouw et al., 2008). These studies indicate that 

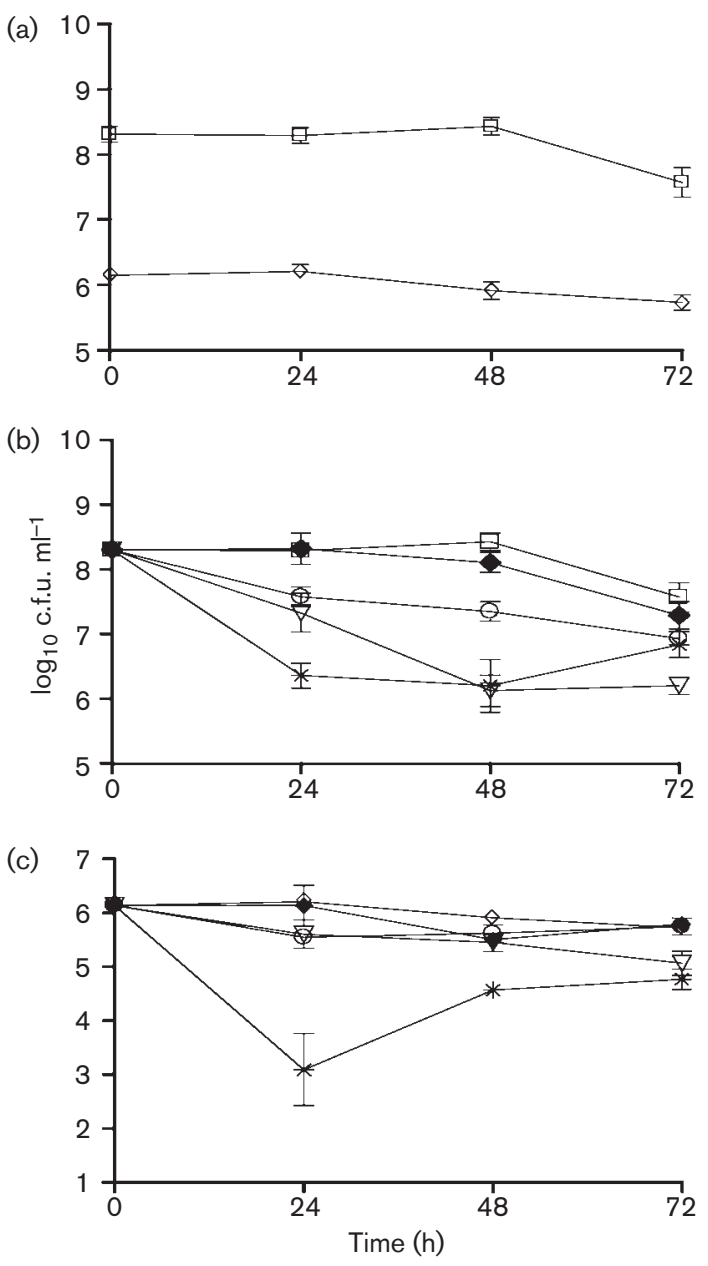

Fig. 4. Relative contributions and viability of $S$. epidermidis and $C$. albicans in polymicrobial biofilms. Polymicrobial biofilms of $S$. epidermidis ATCC 55133 and C. albicans ATCC 32354 were grown in 96-well microtitre plates. The relative contributions of the two organisms (a) were evaluated by sonicating and plating at 0 , 24,48 and 72 h. The $\log _{10}$ c.f.u. $\mathrm{ml}^{-1}$ values are presented as means \pm SEM. Both organisms contributed proportionally to the polymicrobial biofilm composition. Polymicrobial biofilms were exposed to EDTA and NAC at $16 \mathrm{mg} \mathrm{ml}^{-1}$, TLF at $8 \mathrm{mg} \mathrm{ml}^{-1}$, ethanol at $12.5 \%$ or growth medium (control). The viability of $S$. epidermidis ATCC 55133 (b) was significantly decreased $(P<0.05$, two-way ANOVA) by EDTA and ethanol, by NAC at only 24 and $48 \mathrm{~h}$ but not by TLF. The viability of $C$. albicans ATCC 32354 (c) was significantly reduced ( $P<0.05$, two-way ANOVA) only by ethanol and not by EDTA, NAC or TLF. $\square$, Control $S$. epidermidis ATCC 55133; $\diamond$, control C. albicans ATCC 32354; $\nabla$, EDTA $(n=6) ; \bigcirc, \operatorname{NAC}(n=6) ;{ }^{*}$, ethanol $(n=6) ; \diamond, \operatorname{TLF}(n=6)$.

ethanol holds significant promise as an effective and safe catheter-lock solution.

The chelating agent EDTA significantly reduced biofilm viability of $S$. epidermidis in polymicrobial biofilms. In monomicrobial biofilms, EDTA was effective against all $S$. epidermidis and C. albicans strains after 24, 48 and $72 \mathrm{~h}$ of exposure (Supplementary Figs S1 and S2 available with the online journal), which is comparable to other reports. Tetrasodium EDTA treatment $\left(40 \mathrm{mg} \mathrm{ml}^{-1}\right.$ for $21 \mathrm{~h}$ ) significantly reduced the viability of biofilms of $S$. epidermidis, meticillin-resistant $S$. aureus, $P$. aeruginosa, E. coli, K. pneumoniae or C. albicans (Percival et al., 2005). EDTA combined with minocycline has been shown to be effective against biofilms of S. epidermidis, S. aureus and C. albicans in vitro (Raad et al., 2003). The combination of antibiofilm activity and anticoagulant activity makes EDTA a promising candidate for use in catheter-lock solutions against biofilms of common infections.

NAC significantly reduced biofilm viability of S. epidermidis in polymicrobial biofilms at 24 and $48 \mathrm{~h}$. In monomicrobial biofilms, NAC was consistently effective against all strains of S. epidermidis and C. albicans (Supplementary Figs S1 and S2 available with the online journal). NAC $\left(0.007\right.$ to $\left.8 \mathrm{mg} \mathrm{ml}^{-1}\right)$ in combination with fosfomycin (128 and $2000 \mathrm{mg} \mathrm{ml}^{-1}$ ) against four slime-producing uropathogenic E. coli biofilms in vitro and has been shown to reduce EPS and biofilm synthesis (Marchese et al., 2003). Similarly, reduction of EPS (up to $74 \%$ ) and biofilm mass (up to $46 \%$ ) by NAC ( 0.003 to $8 \mathrm{mg} \mathrm{ml}^{-1}$ ) on 15 clinical strains of S. epidermidis biofilms has been reported (Perez-Giraldo et al., 1997).

TLF did not consistently reduce viability of $S$. epidermidis or C. albicans in either polymicrobial or monomicrobial biofilms (Supplementary Figs S1 and S2 available with the online journal). TLF significantly decreased biomass and mean thickness as assessed by confocal microscopy, which may suggest a static effect rather than a microbicidal effect. Singh et al. (2002) demonstrated that subinhibitory concentrations of lactoferrin (10 to $100 \mu \mathrm{g} \mathrm{ml}^{-1}$ ) significantly inhibited the formation of $P$. aeruginosa biofilms in flow chambers. Leitch \& Willcox (1999a) showed that lactoferrin reduced the MIC of vancomycin on biofilms of $S$. epidermidis formed on artificial contact lenses. Iron sequestration by lactoferrin may be responsible for its antimicrobial effect (Singh, 2004; Valenti \& Antonini, 2005). However, TLF on its own may not be an effective catheter-lock solution.

\section{Evaluation of synergy of the antimicrobial combinations by Cls}

Combinations of antimicrobial agents against biofilms may enhance efficacy, reduce drug dosages and minimize the development of drug resistance. Biofilms are inherently resistant to antibiotics, and antimicrobial combinations may be an important strategy for eradicating biofilmassociated infections. Therefore, we evaluated combinations of NAC, EDTA, ethanol and TLF with antibiotics commonly used in neonatology, by discerning inhibitory end points using the XTT assay, similar to the $\mathrm{MBEC}_{50}$ for single agents. Then, we used the median-effects principle (derived from the mass-action law principle) expounded by Chou and colleague (Chou \& Talalay, 1984; Chou, 2006) to evaluate synergy for the antimicrobial combinations. Evaluation of antimicrobial combinations by the 
median-effects method is widely used in cancer and infectious diseases (especially viral diseases) research. The advantages of this method include surmounting the assumption that drug interactions are linear across dosages and drug effects. No general equation fits all the doseresponse curves because mechanisms of drug actions differ. Dose-response curves evaluated at various effective doses (from 50 to $90 \%$ inhibition) may overcome this problem. Therefore, we evaluated drug combinations in a systematic manner at three different effective doses $\left(\mathrm{ED}_{50}, \mathrm{ED}_{75}\right.$ and $\left.E D_{90}\right)$ at equipotency or near equipotency ratios of the drug combinations (Martinez-Irujo et al., 1996).

To examine the validity of applying the median effects equation to antimicrobial susceptibilities using both single drugs and combination of drugs, we calculated $r$, which is the linear coefficient for the goodness of fit of the data to the median effects plot ( $r=1$ indicates perfect conformity). The mean $r$ value for each isolate of S. epidermidis and $C$. albicans, including values for single agents and drug combinations, was $\geqslant 0.95$. This value indicates good conformity of the data to the median effects equation and renders our calculation of CIs valid (Chou, 2006).

\section{Drug combinations with NAC, EDTA, ethanol and TLF indicate synergy}

Against the three strains of S. epidermidis tested (Table 2), EDTA, NAC and ethanol were synergistic with nafcillin and vancomycin $(\mathrm{CI}<1)$ at equipotency or near equipotency ratios at $\mathrm{ED}_{50}, \mathrm{ED}_{75}$ and $\mathrm{ED}_{90}$ with no exceptions. TLF was also synergistic with nafcillin and vancomycin $(\mathrm{CI}<1)$ at equipotency drug-dose ratios at $\mathrm{ED}_{50}, \mathrm{ED}_{75}$ and $\mathrm{ED}_{90}$ with one exception. TLF was not synergistic when combined in a ratio of 250:1 with vancomycin at $\mathrm{ED}_{90}$ against $S$. epidermidis S101. CIs for drug combinations against $S$. epidermidis at constant drug ratios other than equipotency ratios are presented in Supplementary Table S1 (available with the online journal).

Against the two strains of C. albicans tested (Table 3), EDTA and ethanol were synergistic with amphotericin $B$ and fluconazole $(\mathrm{CI}<1)$ at $\mathrm{ED}_{50}, \mathrm{ED}_{75}$ and $\mathrm{ED}_{90}$ with no exceptions. NAC was synergistic with amphotericin $\mathrm{B}$ and fluconazole $(\mathrm{CI}<1)$ in equipotency drug-dose ratios at $\mathrm{ED}_{50}$, $\mathrm{ED}_{75}$ and $\mathrm{ED}_{90}$ with one exception. NAC was not synergistic when combined with fluconazole in a ratio of $1000: 1$ at $\mathrm{ED}_{90}$ against the C. albicans strain ATCC MYA 4441. TLF was synergistic with amphotericin $\mathrm{B}$ and fluconazole $(\mathrm{CI}<1)$ in equipotency drug-dose ratios at $\mathrm{ED}_{50}, \mathrm{ED}_{75}$ and $\mathrm{ED}_{90}$ with one exception. TLF was not synergistic when combined with fluconazole in a ratio of 500:1 (at $\mathrm{ED}_{50}, \mathrm{ED}_{75}$ and $\mathrm{ED}_{90}$ ) against the C. albicans strain ATCC 32354. CIs for drug combinations against $C$. albicans at constant drug ratios other than equipotency ratios are presented in Supplementary Table S2 (available with the online journal).

The mechanisms for the synergistic effects with antifungal and antistaphylococcal agents are speculative and likely to

Table 2. Cls for the drug combinations against $S$. epidermidis biofilms at equipotency ratios

CIs were derived by the median effects principle, and indicate synergy if $\mathrm{CI}<1$, additive effect if $\mathrm{CI}=1$ and antagonism if $\mathrm{CI}>1$. The nonsynergistic ratio is shown in bold. $\mathrm{ED}_{50}, \mathrm{ED}_{75}$, and $\mathrm{ED}_{90}$ represent 50,75 and $90 \%$ inhibitory effects, respectively.

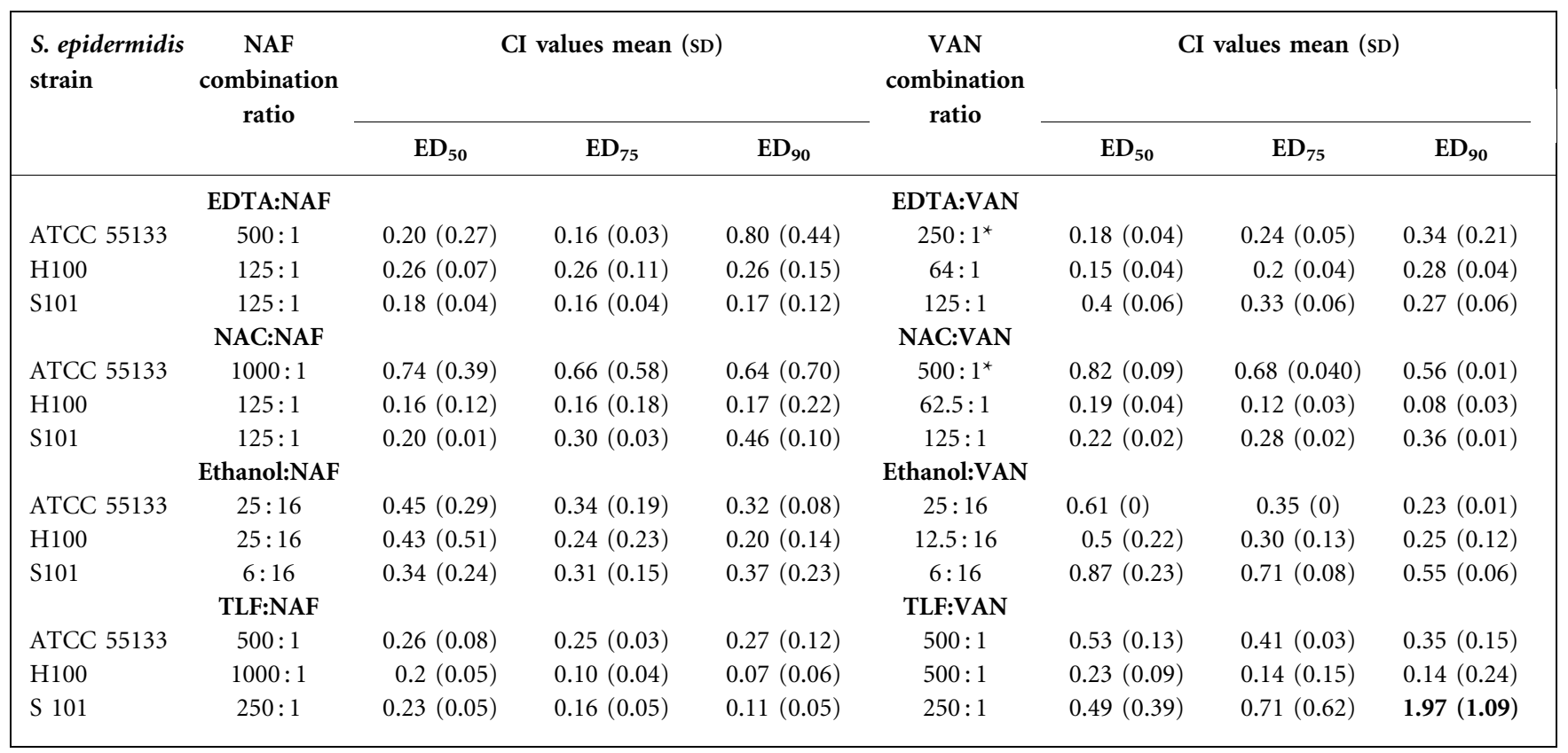

NAF, Nafcillin; VAN, vancomycin.

${ }^{\star}$ Indicates ratios close to equipotency ratios. 
Table 3. Cls for the drug combinations against C. albicans biofilms at equipotency ratios

CIs were derived by the median effects principle, and indicates synergy if $\mathrm{CI}<1$, additive effect if $\mathrm{CI}=1$ and antagonism if $\mathrm{CI}>1$. Non-synergistic combinations are shown in bold. $\mathrm{ED}_{50}, \mathrm{ED}_{75}$, and $\mathrm{ED}_{90}$ represent 50,75 and $90 \%$ inhibitory effects, respectively.

\begin{tabular}{|c|c|c|c|c|c|c|c|c|}
\hline \multirow{2}{*}{$\begin{array}{l}\text { C. albicans } \\
\text { strain }\end{array}$} & \multirow{2}{*}{$\begin{array}{c}\mathrm{AMB} \\
\text { combination } \\
\text { ratio }\end{array}$} & \multicolumn{3}{|c|}{ CI values mean $(\mathrm{SD})$} & \multirow{2}{*}{$\begin{array}{c}\text { FLC } \\
\text { combination } \\
\text { ratio }\end{array}$} & \multicolumn{3}{|c|}{ CI values mean $(\mathrm{SD})$} \\
\hline & & $\mathrm{ED}_{50}$ & $\mathrm{ED}_{75}$ & $\mathrm{ED}_{90}$ & & $\mathrm{ED}_{50}$ & $\mathrm{ED}_{75}$ & $\mathrm{ED}_{90}$ \\
\hline & EDTA:AMB & & & & EDTA:FLC & & & \\
\hline ATCC 32354 & $500: 1$ & $0.14(0.12)$ & $0.11(0.04)$ & $0.10(0.02)$ & $500: 1$ & $0.27(0.13)$ & $0.57(0.39)$ & $2.61(3.89)$ \\
\hline \multirow[t]{2}{*}{$\begin{array}{l}\text { ATCC MYA } \\
4441\end{array}$} & $500: 1$ & $0.07(0.01)$ & $0.03(0.01)$ & $0.01(0.01)$ & $500: 1$ & $0.16(0.07)$ & $0.09(0.09)$ & $0.06(0.08)$ \\
\hline & NAC:AMB & & & & NAC:FLC & & & \\
\hline ATCC 32354 & $500: 1$ & $0.04(0.02)$ & $0.02(0.01)$ & $0.01(0.01)$ & $500: 1$ & $0.08(0.02)$ & $0.07(0.01)$ & $0.05(0.01)$ \\
\hline \multirow{2}{*}{$\begin{array}{l}\text { ATCC MYA } \\
4441\end{array}$} & $1000: 1$ & $0.17(0.09)$ & $0.09(0.01)$ & $0.06(0.04)$ & $1000: 1$ & $0.3(0.11)$ & $0.77(0.26)$ & $1.99(0.61)$ \\
\hline & Ethanol:AMB & & & & Ethanol:FLC & & & \\
\hline ATCC 32354 & $12.5: 1^{\star}$ & $0.51(0.29)$ & $0.40(0.21)$ & $0.37(0.21)$ & $12.5: 1^{\star}$ & $0.61(0.19)$ & $0.54(0.09)$ & $0.51(0.13)$ \\
\hline \multirow{2}{*}{$\begin{array}{l}\text { ATCC MYA } \\
4441\end{array}$} & $12.5: 1 \dagger$ & $0.67(0.36)$ & $0.61(0.11)$ & $0.39(0.15)$ & $12.5: 1 \dagger$ & $0.57(0.27)$ & $0.54(0.16)$ & $0.55(0.09)$ \\
\hline & TLF:AMB & & & & TLF:FLC & & & \\
\hline ATCC 32354 & $500: 1$ & $0.19(0.02)$ & $0.34(0.33)$ & $0.16(0.07)$ & $500: 1$ & $1.02(0.28)$ & $5.34(2.73)$ & $29.93(22.21)$ \\
\hline $\begin{array}{l}\text { ATCC MYA } \\
4441\end{array}$ & $2000: 1$ & $0.11(0.04)$ & $0.06(0.03)$ & $0.04(0.03)$ & $2000: 1$ & $0.08(0.07)$ & $0.10(0.14)$ & $0.18(0.25)$ \\
\hline
\end{tabular}

AMB, Amphotericin B; FLC, fluconazole.

${ }^{\star}$ Ratios not at equipotency but combinations of $\mathrm{MBEC}_{50}$ of ethanol with $1 / 64$ th or $1 / 32 \mathrm{nd} \mathrm{MBEC}_{50}$ concentrations of amphotericin $\mathrm{B}$ and fluconazole, respectively.

$\dagger$ Ratios not at equipotency but combinations of $\mathrm{MBEC}_{50}$ of ethanol with $1 / 64$ th or $1 / 32$ nd $\mathrm{MBEC}_{50}$ concentrations of amphotericin $\mathrm{B}$ and fluconazole, respectively.

vary with the different agents. EDTA is a divalent cation (calcium and magnesium) chelator and may have inhibitory effects against Candida and Staphylococcus strains (Gordon et al., 1991; Ozerdem Akpolat et al., 2003; Percival et al., 2005; Ramage et al., 2007; Root et al., 1988). NAC and ethanol may act by inhibiting the production of EPS or facilitating its degradation (Alem \& Douglas, 2005; Marchese et al., 2003; Olofsson et al., 2003; Raad et al., 2007a). The synergistic effects of lactoferrin may be due to iron sequestering effects or a direct lytic effect on the cell membranes of Candida and Staphylococcus (Leitch \& Willcox, 1999b; Xu et al., 1999). We have reported the synergistic effects of human recombinant lactoferrin on planktonic cells of clinical isolates of $S$. epidermidis and $C$. albicans (Venkatesh \& Liang, 2008). We speculate that the action of lactoferrin on cell membranes complements the action of other drugs.

The limitations of this study follow the inherent variability in antimicrobial susceptibility testing by broth microdilution method and chequerboard assays. Biological replicates were performed on two different days. Replicates on the same experimental day (same experimental conditions) were averaged, generating two sets of data for analyses. Chequerboard combinations used twofold serial dilutions of the drug, and the effects of the drug concentrations between the twofold dilutions were not evaluated. Our results need to be validated on biofilms formed on catheter material and under flow conditions that exist in vivo. We intend to pursue this research on biofilms formed in flow devices such as the Robbins flow device, the Stovall flow cell system or other flow cell systems in future follow-up studies. The general limitation of experiments in vitro in terms of correlation to effects in vivo exist, and may be due to the environment and physical milieu as well as the participation of host defence mechanisms. Catheter-lock antimicrobial combinations need to be evaluated in appropriate animal models of catheter infections (Andes et al., 2004; Schinabeck et al., 2004) using clinical isolates of organisms of interest.

The key observations of this study are that EDTA, NAC, ethanol and TLF decreased the biomass and thickness of $S$. epidermidis and $C$. albicans biofilms. EDTA, NAC and ethanol consistently reduced viability in monomicrobial biofilms. Ethanol $\left(12.5 \%, \mathrm{MBEC}_{50}\right)$ was the only agent that reduced viability of $C$. albicans in polymicrobial biofilms. Ethanol at higher concentrations (25 to $70 \%$ ) has been found to be effective against biofilms in other studies (Chambers et al., 2006; Raad et al., 2007a). NAC, EDTA, ethanol and TLF were synergistic with antibacterial and antifungal antibiotics against biofilms of S. epidermidis and 
C. albicans. Polymicrobial infections account for approximately half of all paediatric CRBSIs resulting from catheter biofilms. Candida (approximately 10\%) and Gram-positive organisms (mostly staphylococci, approximately $10 \%)$ are other major causes of paediatric CRBSIs (Almuneef et al., 2006). Precious vascular indwelling catheters are removed after refractory $C$. albicans CRBSI and S. epidermidis CRBSIs. Our results suggest that NAC, EDTA or ethanol alone or in combination with antibiotics used as catheter-lock solutions for 24 to $72 \mathrm{~h}$ may eradicate infections due to S. epidermidis and C. albicans and help in the retention of vascular catheters. We conclude that catheter-lock solutions utilizing NAC, EDTA and ethanol individually or in combination with antibiotics might be used to salvage infected catheters, and will directly impact patient morbidity and health-care costs.

\section{ACKNOWLEDGEMENTS}

We acknowledge the help of Agennix Inc. for donating recombinant human lactoferrin (TLF) for our research. We thank Claus Sternberg $\mathrm{PhD}$, BioSys-DTU, Denmark, for the COMSTAT 2 software used in the analyses of confocal images of biofilms. We thank Barbara Trautner MD, VA Center, Houston, TX (for confocal imaging of biofilms), Sara Jones PhD and members of J. Versalovic's lab for insightful discussions.

\section{REFERENCES}

Alem, M. A. \& Douglas, L. J. (2005). Prostaglandin production during growth of Candida albicans biofilms. J Med Microbiol 54, 1001-1005.

Almuneef, M. A., Memish, Z. A., Balkhy, H. H., Hijazi, O., Cunningham, G. \& Francis, C. (2006). Rate, risk factors and outcomes of catheter-related bloodstream infection in a paediatric intensive care unit in Saudi Arabia. J Hosp Infect 62, 207-213.

Anaissie, E., Samonis, G., Kontoyiannis, D., Costerton, J., Sabharwal, U., Bodey, G. \& Raad, I. (1995). Role of catheter colonization and infrequent hematogenous seeding in catheter-related infections. Eur J Clin Microbiol Infect Dis 14, 134-137.

Andes, D., Nett, J., Oschel, P., Albrecht, R., Marchillo, K. \& Pitula, A. (2004). Development and characterization of an in vivo central venous catheter Candida albicans biofilm model. Infect Immun 72, 6023-6031.

Bachmann, S. P., Ramage, G., VandeWalle, K., Patterson, T. F., Wickes, B. L. \& Lopez-Ribot, J. L. (2003). Antifungal combinations against Candida albicans biofilms in vitro. Antimicrob Agents Chemother 47, 3657-3659.

Broom, J., Woods, M., Allworth, A., McCarthy, J., Faoagali, J., Macdonald, S. \& Pithie, A. (2008). Ethanol lock therapy to treat tunnelled central venous catheter-associated blood stream infections: results from a prospective trial. Scand J Infect Dis 40, 399-406.

Cerca, N., Pier, G. B., Vilanova, M., Oliveira, R. \& Azeredo, J. (2004). Influence of batch or fed-batch growth on Staphylococcus epidermidis biofilm formation. Lett Appl Microbiol 39, 420-424.

Cerca, N., Martins, S., Cerca, F., Jefferson, K. K., Pier, G. B., Oliveira, R. \& Azeredo, J. (2005). Comparative assessment of antibiotic susceptibility of coagulase-negative staphylococci in biofilm versus planktonic culture as assessed by bacterial enumeration or rapid XTT colorimetry. J Antimicrob Chemother 56, 331-336.
Chambers, S. T., Peddie, B. \& Pithie, A. (2006). Ethanol disinfection of plastic-adherent micro-organisms. J Hosp Infect 63, 193-196.

Chou, T. C. (2006). Theoretical basis, experimental design, and computerized simulation of synergism and antagonism in drug combination studies. Pharmacol Rev 58, 621-681.

Chou, T. C. \& Talalay, P. (1984). Quantitative analysis of dose-effect relationships: the combined effects of multiple drugs or enzyme inhibitors. Adv Enzyme Regul 22, 27-55.

CLSI (2002). Reference Method for Broth Dilution Antifungal Susceptibility Testing of Yeasts, approved standard, 2nd edn, M27A2. Wayne, PA: CLSI.

CLSI (2003). Methods for Dilution Antimicrobial Susceptibility Tests for Bacteria that Grow Aerobically, approved standard, 6th edn, M7-A7. Wayne, PA: CLSI.

CLSI (2007). Performance Standards for Antimicrobial Susceptibility Testing, 17th informational supplement, M100-S17.27. Wayne, PA: CLSI.

Console, G., Calabro, C., Nardulli, P., Digiuseppe, F., Rucci, A., Russo, P. \& Geppetti, P. (2007). Clinical and economic effects of central venous catheters on oncology patient care. J Chemother 19, 309-314.

Crnich, C. J., Halfmann, J. A., Crone, W. C. \& Maki, D. G. (2005). The effects of prolonged ethanol exposure on the mechanical properties of polyurethane and silicone catheters used for intravascular access. Infect Control Hosp Epidemiol 26, 708-714.

Dimick, J. B., Pelz, R. K., Consunji, R., Swoboda, S. M., Hendrix, C. W. \& Lipsett, P. A. (2001). Increased resource use associated with catheter-related bloodstream infection in the surgical intensive care unit. Arch Surg 136, 229-234.

Domingue, G., Ellis, B., Dasgupta, M. \& Costerton, J. W. (1994). Testing antimicrobial susceptibilities of adherent bacteria by a method that incorporates guidelines of the National Committee for Clinical Laboratory Standards. J Clin Microbiol 32, 2564-2568.

Donelli, G. (2006). Vascular catheter-related infection and sepsis. Surg Infect (Larchmt) 7 (Suppl. 2), S25-S27.

Faix, R. G. \& Kovarik, S. M. (1989). Polymicrobial sepsis among intensive care nursery infants. J Perinatol 9, 131-136.

Furuya, E. Y. \& Lowy, F. D. (2003). Antimicrobial strategies for the prevention and treatment of cardiovascular infections. Curr Opin Pharmacol 3, 464-469.

Gandelman, G., Frishman, W. H., Wiese, C., Green-Gastwirth, V., Hong, S., Aronow, W. S. \& Horowitz, H. W. (2007). Intravascular device infections: epidemiology, diagnosis, and management. Cardiol Rev 15, 13-23.

Gil, M. L., Casanova, M. \& Martinez, J. P. (1994). Changes in the cell wall glycoprotein composition of Candida albicans associated to the inhibition of germ tube formation by EDTA. Arch Microbiol 161, 489494.

Gordon, C. A., Hodges, N. A. \& Marriott, C. (1991). Use of slime dispersants to promote antibiotic penetration through the extracellular polysaccharide of mucoid Pseudomonas aeruginosa. Antimicrob Agents Chemother 35, 1258-1260.

Hawser, S. P., Norris, H., Jessup, C. J. \& Ghannoum, M. A. (1998). Comparison of a 2,3-bis(2-methoxy-4-nitro-5-sulfophenyl)-5[(phenylamino)carbonyl]-2H-tetrazolium hydroxide (XTT) colorimetric method with the standardized National Committee for Clinical Laboratory Standards method of testing clinical yeast isolates for susceptibility to antifungal agents. J Clin Microbiol 36, 1450-1452.

Heydorn, A., Nielsen, A. T., Hentzer, M., Sternberg, C., Givskov, M., Ersboll, B. K. \& Molin, S. (2000). Quantification of biofilm structures 
by the novel computer program COMSTAT. Microbiology 146, 23952407.

Karlowicz, M. G., Hashimoto, L. N., Kelly, R. E., Jr \& Buescher, E. S. (2000). Should central venous catheters be removed as soon as candidemia is detected in neonates? Pediatrics 106, E63.

Kaufman, D. \& Fairchild, K. D. (2004). Clinical microbiology of bacterial and fungal sepsis in very-low-birth-weight infants. Clin Microbiol Rev 17, 638-680.

Leitch, E. C. \& Willcox, M. D. (1999a). Lactoferrin increases the susceptibility of $S$. epidermidis biofilms to lysozyme and vancomycin. Curr Eye Res 19, 12-19.

Leitch, E. C. \& Willcox, M. D. (1999b). Elucidation of the antistaphylococcal action of lactoferrin and lysozyme. $J \mathrm{Med}$ Microbiol 48, 867-871.

Levy, S. B. (1998). Antimicrobial resistance: bacteria on the defence. Resistance stems from misguided efforts to try to sterilise our environment. BMJ 317, 612-613.

Marchese, A., Bozzolasco, M., Gualco, L., Debbia, E. A., Schito, G. C. \& Schito, A. M. (2003). Effect of fosfomycin alone and in combination with $\mathrm{N}$-acetylcysteine on E. coli biofilms. Int J Antimicrob Agents 22 (Suppl. 2), 95-100.

Martinez-Irujo, J. J., Villahermosa, M. L., Alberdi, E. \& Santiago, E. (1996). A checkerboard method to evaluate interactions between drugs. Biochem Pharmacol 51, 635-644.

McKenzie, F. E. (2006). Case mortality in polymicrobial bloodstream infections. J Clin Epidemiol 59, 760-761.

Mouw, E., Chessman, K., Lesher, A. \& Tagge, E. (2008). Use of an ethanol lock to prevent catheter-related infections in children with short bowel syndrome. J Pediatr Surg 43, 1025-1029.

Noyola, D. E., Fernandez, M., Moylett, E. H. \& Baker, C. J. (2001). Ophthalmologic, visceral, and cardiac involvement in neonates with candidemia. Clin Infect Dis 32, 1018-1023.

O’Grady, N. P., Alexander, M., Dellinger, E. P., Gerberding, J. L., Heard, S. O., Maki, D. G., Masur, H., McCormick, R. D., Mermel, L. A. \& other authors (2002). Guidelines for the prevention of intravascular catheter-related infections. Am J Infect Control 30, 476-489.

Olofsson, A. C., Hermansson, M. \& Elwing, H. (2003). N-Acetyl-Lcysteine affects growth, extracellular polysaccharide production, and bacterial biofilm formation on solid surfaces. Appl Environ Microbiol 69, 4814-4822.

Ozerdem Akpolat, N., Elci, S., Atmaca, S., Akbayin, H. \& Gul, K. (2003). The effects of magnesium, calcium and EDTA on slime production by Staphylococcus epidermidis strains. Folia Microbiol (Praha) 48, 649-653.

Pawar, M., Mehta, Y., Kapoor, P., Sharma, J., Gupta, A. \& Trehan, N. (2004). Central venous catheter-related blood stream infections: incidence, risk factors, outcome, and associated pathogens. J Cardiothorac Vasc Anesth 18, 304-308.

Percival, S. L., Kite, P., Eastwood, K., Murga, R., Carr, J., Arduino, M. J. \& Donlan, R. M. (2005). Tetrasodium EDTA as a novel central venous catheter lock solution against biofilm. Infect Control Hosp Epidemiol 26, 515-519.

Perez-Giraldo, C., Rodriguez-Benito, A., Moran, F. J., Hurtado, C., Blanco, M. T. \& Gomez-Garcia, A. C. (1997). Influence of Nacetylcysteine on the formation of biofilm by Staphylococcus epidermidis. J Antimicrob Chemother 39, 643-646.
Raad, I. I. \& Hanna, H. A. (2002). Intravascular catheter-related infections: new horizons and recent advances. Arch Intern Med 162, 871-878.

Raad, I., Chatzinikolaou, I., Chaiban, G., Hanna, H., Hachem, R., Dvorak, T., Cook, G. \& Costerton, W. (2003). In vitro and ex vivo activities of minocycline and EDTA against microorganisms embedded in biofilm on catheter surfaces. Antimicrob Agents Chemother 47, 3580-3585.

Raad, I., Hanna, H., Dvorak, T., Chaiban, G. \& Hachem, R. (2007a). Optimal antimicrobial catheter lock solution, using different combinations of minocycline, EDTA, and 25-percent ethanol, rapidly eradicates organisms embedded in biofilm. Antimicrob Agents Chemother 51, 78-83.

Raad, I., Hanna, H., Jiang, Y., Dvorak, T., Reitzel, R., Chaiban, G., Sherertz, R. \& Hachem, R. (2007b). Comparative activities of daptomycin, linezolid, and tigecycline against catheter-related methicillin-resistant Staphylococcus bacteremic isolates embedded in biofilm. Antimicrob Agents Chemother 51, 1656-1660.

Ramage, G. \& Lopez-Ribot, J. L. (2005). Techniques for antifungal susceptibility testing of Candida albicans biofilms. Methods Mol Med 118, 71-79.

Ramage, G., Wickes, B. L. \& Lopez-Ribot, J. L. (2007). Inhibition on Candida albicans biofilm formation using divalent cation chelators (EDTA). Mycopathologia 164, 301-306.

Rello, J., Ochagavia, A., Sabanes, E., Roque, M., Mariscal, D., Reynaga, E. \& Valles, J. (2000). Evaluation of outcome of intravenous catheter-related infections in critically ill patients. Am J Respir Crit Care Med 162, 1027-1030.

Root, J. L., McIntyre, O. R., Jacobs, N. J. \& Daghlian, C. P. (1988). Inhibitory effect of disodium EDTA upon the growth of Staphylococcus epidermidis in vitro: relation to infection prophylaxis of Hickman catheters. Antimicrob Agents Chemother 32, 1627-1631.

Schinabeck, M. K., Long, L. A., Hossain, M. A., Chandra, J., Mukherjee, P. K., Mohamed, S. \& Ghannoum, M. A. (2004). Rabbit model of Candida albicans biofilm infection: liposomal amphotericin B antifungal lock therapy. Antimicrob Agents Chemother 48, 17271732.

Singh, P. K. (2004). Iron sequestration by human lactoferrin stimulates $P$. aeruginosa surface motility and blocks biofilm formation. Biometals 17, 267-270.

Singh, P. K., Parsek, M. R., Greenberg, E. P. \& Welsh, M. J. (2002). A component of innate immunity prevents bacterial biofilm development. Nature 417, 552-555.

Stoodley, P., Wilson, S., Hall-Stoodley, L., Boyle, J. D., Lappin-Scott, H. M. \& Costerton, J. W. (2001). Growth and detachment of cell clusters from mature mixed-species biofilms. Appl Environ Microbiol 67, 5608-5613.

Valenti, P. \& Antonini, G. (2005). Lactoferrin: an important host defence against microbial and viral attack. Cell Mol Life Sci 62, 25762587.

Venkatesh, M. P. \& Liang, R. (2008). Human recombinant lactoferrin is synergistic with antimicrobials commonly used in neonatal practice against coagulase-negative staphylococci and Candida albicans causing neonatal sepsis. J Med Microbiol 57, 1113-1121.

Xu, Y. Y., Samaranayake, Y. H., Samaranayake, L. P. \& Nikawa, H. (1999). In vitro susceptibility of Candida species to lactoferrin. Med Mycol 37, 35-41. 